\title{
LINC01355 suppresses breast cancer growth through FOXO3-mediated transcriptional repression of CCND1
}

\author{
Bolun Ai ${ }^{1}$, Xiangyi Kong ${ }^{1}$, Xiangyu Wang ${ }^{1}$, Kai Zhang ${ }^{2}$, Xue Yang ${ }^{1}$, Jie Zhai ${ }^{1}$, Ran Gao ${ }^{1}$, Yihang Qi ${ }^{1}$, Jing Wang ${ }^{1}$, \\ Zhongzhao Wang ${ }^{1}$ and Yi Fang ${ }^{1}$
}

\begin{abstract}
Previously, several protein-coding tumor suppressors localized at 1p36 have been reported. In the present work, we focus on functional long non-coding RNAs (IncRNAs) embedded in this locus. Small interfering RNA was used to identify IncRNA candidates with growth-suppressive activities in breast cancer. The mechanism involved was also explored. LINC01355 were downregulated in breast cancer cells relative to non-malignant breast epithelial cells. Overexpression of LINC01355 significantly inhibited proliferation, colony formation, and tumorigenesis of breast cancer cells. LINC01355 arrested breast cancer cells at the G0/G1 phase by repressing CCND1. Moreover, LINC01355 interacted with and stabilized FOXO3 protein, leading to transcriptional repression of CCND1. Importantly, LINC01355-mediated suppression of breast cancer growth was reversed by knockdown of FOXO3 or overexpression of CCND1. Clinically, LINC01355 was downregulated in breast cancer specimens and correlated with more aggressive features. There was a negative correlation between LINC01355 and CCND1 expression in breast cancer samples. LINC01355 acts as a tumor suppressor in breast cancer, which is ascribed to enhancement of FOXO3-mediated transcriptional repression of CCND1. Re-expression of LINC01355 may provide a potential therapeutic strategy to block breast cancer growth and progression.
\end{abstract}

\section{Introduction}

Breast cancer is the most common malignancy in females ${ }^{1}$. Despite noticeable advancement in detection and treatment, the disease is still devastating ${ }^{2,3}$. Understanding the molecular basis for the aggressive phenotype of breast cancer is critical to identify novel targets to improve therapeutic outcomes.

Correspondence: Jing Wang (wangjing@cicams.ac.cn) or

Zhongzhao Wang (wangzhongzhao206@sina.com) or

Yi Fang (fangyi0501@vip.sina.com) (fangyi@cicams.ac.cn)

'Department of Breast Surgical Oncology, National Cancer Center/National

Clinical Research Center for Cancer/Cancer Hospital, Chinese Academy of

Medical Sciences and Peking Union Medical College, Beijing, China

${ }^{2}$ Department of Cancer Prevention, National Cancer Center/National Clinica

Research Center for Cancer/Cancer Hospital, Chinese Academy of Medical

Sciences and Peking Union Medical College, Beijing, China

These authors contributed equally: Bolun Ai, Xiangyi Kong, Xiangyu Wang,

Kai Zhang

Edited by E. Baehrecke
Abnormality of the chromosome $1 \mathrm{p} 36$ locus has been detected in many human cancers ${ }^{4-6}$. Nagai et al. ${ }^{7}$ reported a common deletion in the $1 \mathrm{p} 36$ region in breast cancer patients $(10 / 44 ; 23 \%)$, suggesting that the region contains potential antitumor genes. Previously, a number of $1 \mathrm{p} 36$ genes have been identified as tumor suppressors, such as CAMTA1, AJAP1, and CASZ1 ${ }^{8-10}$. Apart from the protein-coding genes, tumor suppressive non-coding genes are also presented in the $1 \mathrm{p} 36$ locus 6 . For instance, microRNA (miR)-34a, which is located in $1 \mathrm{p} 36.23$, has a pro-apoptotic role in neuroblastoma cells ${ }^{11}$.

Long non-coding RNAs (lncRNAs) are a large class of transcripts of longer than $200 \mathrm{bp}$ and regulate a variety of biological processes, including cell proliferation, motility, and invasion ${ }^{12}$. Accumulating evidence has indicated that lncRNAs are involved in tumor development and

\section{(c) The Author(s) 2019}

(c) (i) Open Access This article is licensed under a Creative Commons Attribution 4.0 International License, which permits use, sharing, adaptation, distribution and reproduction cc) in any medium or format, as long as you give appropriate credit to the original author(s) and the source, provide a link to the Creative Commons license, and indicate if changes were made. The images or other third party material in this article are included in the article's Creative Commons license, unless indicated otherwise in a credit line to the material. If material is not included in the article's Creative Commons license and your intended use is not permitted by statutory regulation or exceeds the permitted use, you will need to obtain permission directly from the copyright holder. To view a copy of this license, visit http://creativecommons.org/licenses/by/4.0/. 
progression $^{13}$. For instance, lncRNA SNHG14 was found to confer trastuzumab resistance to breast cancer cells ${ }^{14}$. lncRNA MIR100HG shows the ability to promote breast cancer cell proliferation ${ }^{15}$. IncRNAs can regulate gene expression through multiple mechanisms. It has been documented that lncRNA can sponge specific miRs and indirectly modulate gene expression at the posttranscriptional level ${ }^{16}$. Alternatively, lncRNAs can interact with transcription factors or epigenetic proteins to regulate target gene transcription ${ }^{14,17}$.

Forkhead box O3 (FOXO3) belongs to the O subclass of the forkhead family of transcription factors and is a wellestablished tumor suppressor ${ }^{18}$. FOXO3 has been shown to control various aspects of breast cancer biology ${ }^{19-21}$. Song et al. ${ }^{19}$ reported that restoration of FOXO3 induces apoptosis and cell cycle arrest and reverses 5-fluorouracil resistance in human breast cancer cells. Sisci et al. ${ }^{20}$ demonstrated that overexpression of FOXO3 decreases migration, invasion, and anchorage-independent growth of estrogen receptor $\alpha$-positive breast cancer cells. Multiple target genes such as CCND1 and VEGF have been proposed to mediate the anticancer activity of $\mathrm{FOXO}^{21,22}$.

Although many lncRNA transcripts encoded by the 1 p36 locus have been deposited in GenBank database, their biological functions have not been deciphered yet. In this study, we used small interfering RNA (siRNA) technology to deplete IncRNA candidates transcribed from 1 p36 in an immortal, nontransformed breast epithelial cell line, MCF10A, with the aim to identify novel tumorsuppressing lncRNAs. The expression and function of the interested lncRNA(s) in breast cancer was explored.

\section{Results}

\section{LINC01355 functions as a tumor suppressor}

In this study, we included 37 candidate lncRNAs that are transcribed from the $1 \mathrm{p} 36$ locus. Among them, LINC01342, PINK1-AS, PIK3CD-AS1, LINC01772, and LINC01355 were downregulated in MCF7 cells compared with MCF10A cells (Supplementary Figure S1). Specific siRNAs were used to deplete the five lncRNAs in MCF10A cells. Of note, knockdown of LINC01355 (Fig. 1a) led to a significant increase in the proliferation of MCF10A cells (Fig. 1b). However, depletion of the other lncRNAs tested had minimal effect on the proliferation of MCF10A cells (data not shown). As breast cancer cell lines showed a general downregulation of LINC01355 (Fig. 1c), we performed LINC01355 gain-of-function studies in both MCF7 and MDA-MB-231 cells. We found that overexpression of LINC01355 (Fig. 1d) significantly inhibited the proliferation (Fig. 1e) and colony formation (Fig. 1f) of breast cancer cells. Several studies have indicated that multiple pathways including PI3K/Akt, ERK, and STAT3 are engaged in breast cancer growth ${ }^{23-25}$. Next, we examined the effect of LINC01355 overexpression on the activation of PI3K/Akt, ERK, and STAT3 signaling. The results showed that ectopic expression of LINC01355 did not change the activation status of PI3K/Akt, ERK, or STAT3 (Supplementary Figure S2).

\section{LINC01355 overexpression suppresses breast cancer cell growth in vivo}

Next, we tested whether LINC01355 can antagonize the tumorigenesis of breast cancer cells in vivo. To address this, LINC01355-overexpressing and control MCF7 cells were inoculated into female nude mice, and tumor growth was monitored. We noted that the xenograft tumor growth was significantly reduced in the LINC 01355 group compared with the control group $(P<0.05$; Fig. 2 a). Also, final tumor weight was $68 \%$ lower in the LINC01355 group than that in the control group $(P<0.05$; Fig. $2 b)$. Consistent with this, tumor tissues from LINC01355overexpressing cells had reduced proliferation (Fig. 2c and d), as determined by immunohistochemical analysis. Taken together, LINC01355 acts as a tumor suppressor in breast cancer.

\section{LINC01355 induces cell cycle arrest at the G0/G1 phase by repressing CCND1 expression}

Next, we explored the effect of LINC01355 dysregulation on cell cycle progression. Of note, LINC01355overexpressing MCF7 (Fig. 3a) and MDA-MB-231 (Fig. 3b) cells exhibited an accumulation at the G0/G1 phase and concomitant reduction at the $\mathrm{S}$ phase, indicating G0/G1 cell cycle arrest. Real-time PCR analysis of cell cycle related genes revealed that CCND1 expression was reduced in LINC01355-overexpressing cells relative to control cells (Fig. 3c). Consistent with the reduction of CCND1 transcripts, we also found a decline in the cyclin D1 protein level after LINC01355 overexpression (Fig. 3d). However, the other genes tested, i.e., CCND3, CCNE2, CDK4, CDK6, CDKN1A, and CDKN1B, were unaffected by LINC01355 (Supplementary Figure S3).

To validate the hypothesis that LINC01355-mediated cell cycle arrest is mainly ascribed to reduced cyclin D1 levels, we performed rescue experiments by overexpressing cyclin D1. As a result, enforced expression of cyclin D1 (Fig. 3e) significantly reversed LINC01355 overexpression-induced changes in cell cycle distribution in MCF7 and MDA-MB-231 cells (Fig. 3f). These results indicate cyclin D1 as a critical mediator for the anticancer activity of LINC01355.

\section{LINC01355 interacts with and stabilizes $\mathrm{FOXO}$ protein}

To uncover the detailed mechanism underlying the action of LINC01355, we conducted RNA pull-down assays in MCF7 cell extracts followed by proteomic analysis of LINC01355-associated proteins. The results showed that a number of proteins were highly enriched in 

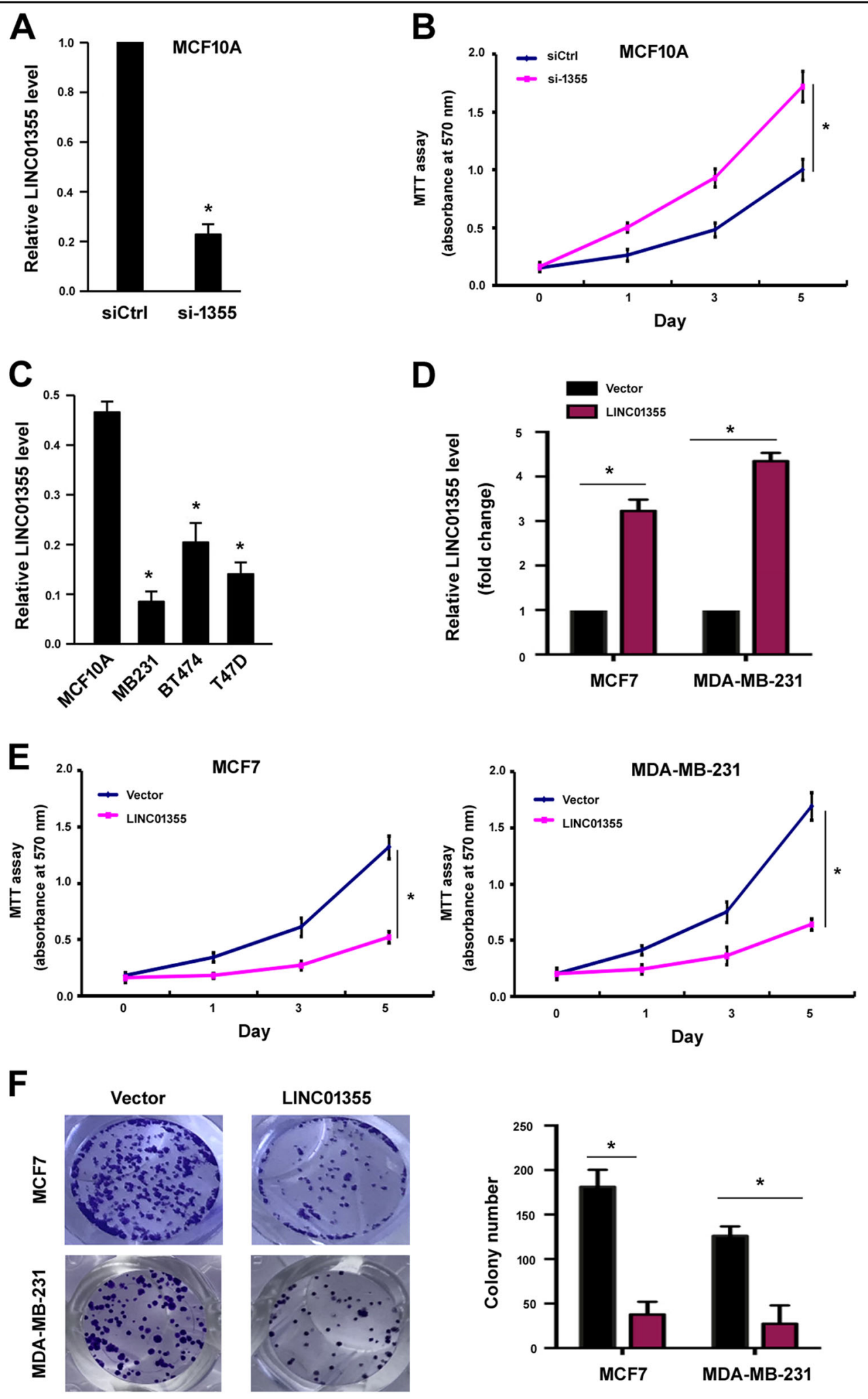

Fig. 1 LINC01355 is downregulated and suppresses cell growth in breast cancer cells. a LINC01355 expression was detected by qRT-PCR in MCF10A cells transfected with control siRNA (siCtrl) or LINC01355-targeting siRNA (si-1355). b Cell proliferation was determined by the MTT assays at indicated time points. c LINC01355 expression was detected by qRT-PCR in indicated cell lines. $\mathbf{d}$ LINC01355 expression was detected by qRT-PCR in MCF7 and MDA-MB-231 cells transfected with empty vector or LINC01355-expressing plasmid. e Cell proliferation was determined by the MTT assays at indicated time points. $\mathbf{f}$ Colony formation assay was performed to evaluate the colony formation ability of MCF7 and MDA-MB-231 cells transfected with indicated constructs. Left, representative images of colonies stained with crystal violet. ${ }^{*} P<0.05$ 


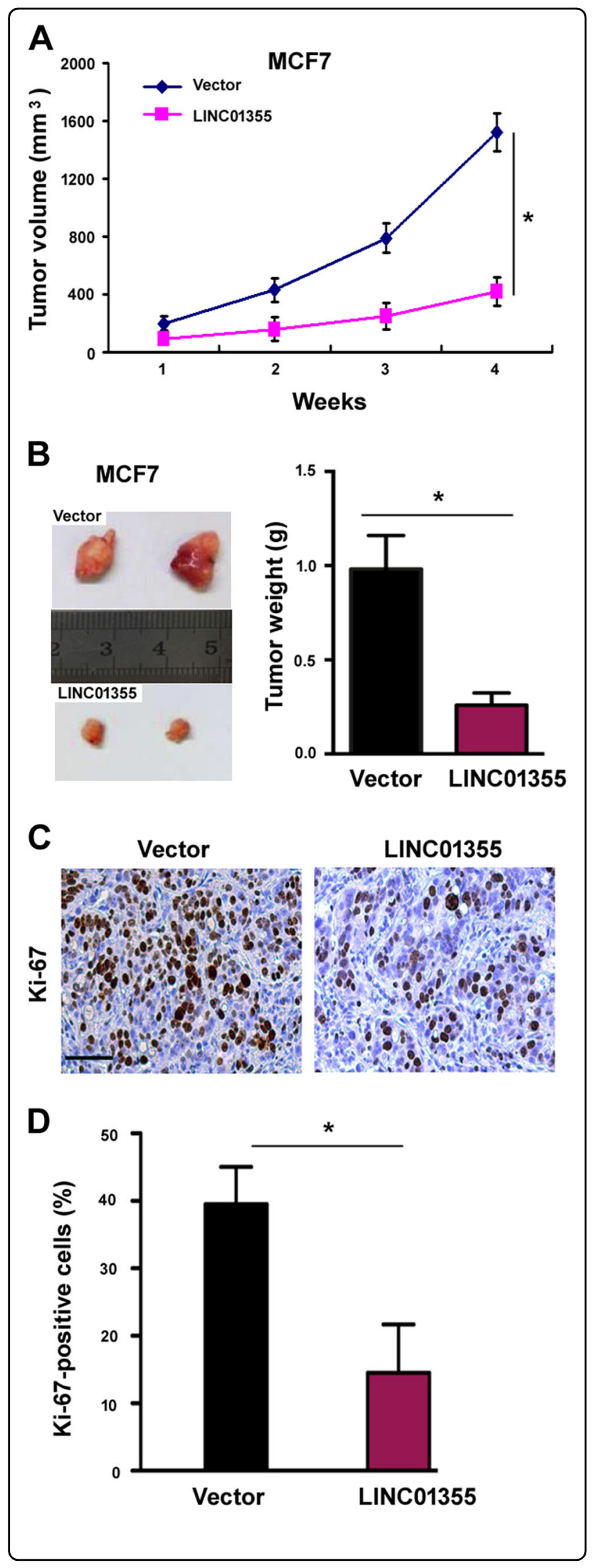

Fig. 2 LINC01355 overexpression suppresses breast cancer cell growth in vivo. a MCF7 cells were stably transfected with empty vector or LINC01355-expressing plasmid and inoculated into nude mice. Growth curves were plotted based on tumor volume. $\mathbf{b}$ Left, representative images of xenograft tumors. Right, final tumor weight was determined 4 weeks after cell injection. c Representative images of Ki-67 immunostaining in tumor samples. Scale bar $=100 \mu \mathrm{m}$. d Quantification of Ki-67 positive cells for each group. ${ }^{*} P<0.05$

the LINC01355 pull-down complexes. Among them, FOXO3 attracted more attention because of its broadspectrum antitumor activity ${ }^{19,20}$. Western blot analysis confirmed the presence of FOXO3 in the complex pulled down by biotinylated LINC01355 sense transcript but not by antisense transcript (Fig. 4a). To validate the association between LINC01355 and FOXO3, radioimmunoprecipitation (RIP) assay was further performed. We found that LINC01355 could be detected in FOXO3 immunoprecipitates from MCF7 cells (Fig. 4b).

Next, we examined the effect of LINC01355 on the expression of FOXO3 in breast cancer cells. Notably, overexpression of LINC01355 caused an elevation in the protein level of FOXO3 in both MCF7 and MDA-MB-231 cells (Fig. 4c). However, the mRNA level of FOXO3 remained unchanged (Fig. 4d). We speculated that LINC01355-mediated upregulation of FOXO3 protein may be a consequence of enhanced protein stability. In support of this hypothesis, the half-life of FOXO3 protein was longer in LINC01355-overexpressing MCF7 cells than that in control cells (Fig. 4e). In contrast, knockdown of LINC01355 diminished the level of FOXO3 protein (Fig. 4f) and accelerated FOXO3 protein turnover (Fig. 4g). These data indicate that LINC01355 acts as a stabilizer of FOXO3 protein.

\section{LINC01355 promotes FOXO3-mediated transcriptional repression of CCND1}

Given that FOXO3 is a transcriptional repressor of $\mathrm{CCND}^{21}$, we next checked whether FOXO3 is involved in LINC01355-dependent reduction of CCND1 in breast cancer cells. To this end, we performed FOXO3 knockdown experiments (Fig. 5a). We found that LINC01355induced downregulation of CCND1 was remarkably impaired when FOXO3 was depleted (Fig. 5b). Moreover, re-expression of FOXO3 (Fig. 5a) restored LINC01355mediated inhibition of CCND1 expression (Fig. 5b). Chromatin immunoprecipitation (ChIP) assay further demonstrated that LINC01355 overexpression led to a selective enrichment of FOXO3 protein at the promoter of CCND1 (Fig. 5c and d). As a control, the FOXO3 binding to the VEGF promoter was not altered by LINC01355 overexpression (Fig. 5c and d). Taken 

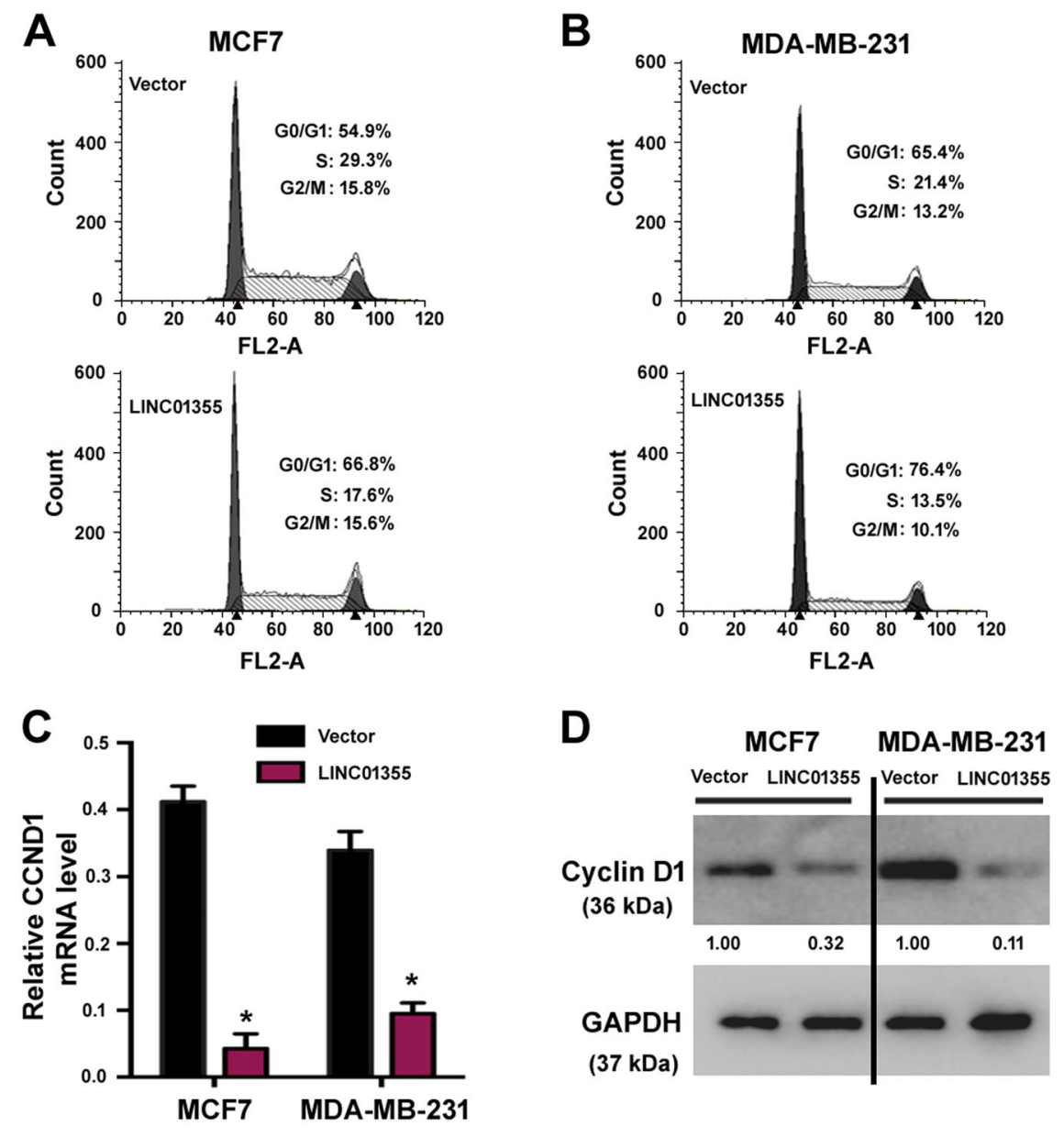

$\mathbf{E}$

$\mathbf{F}$
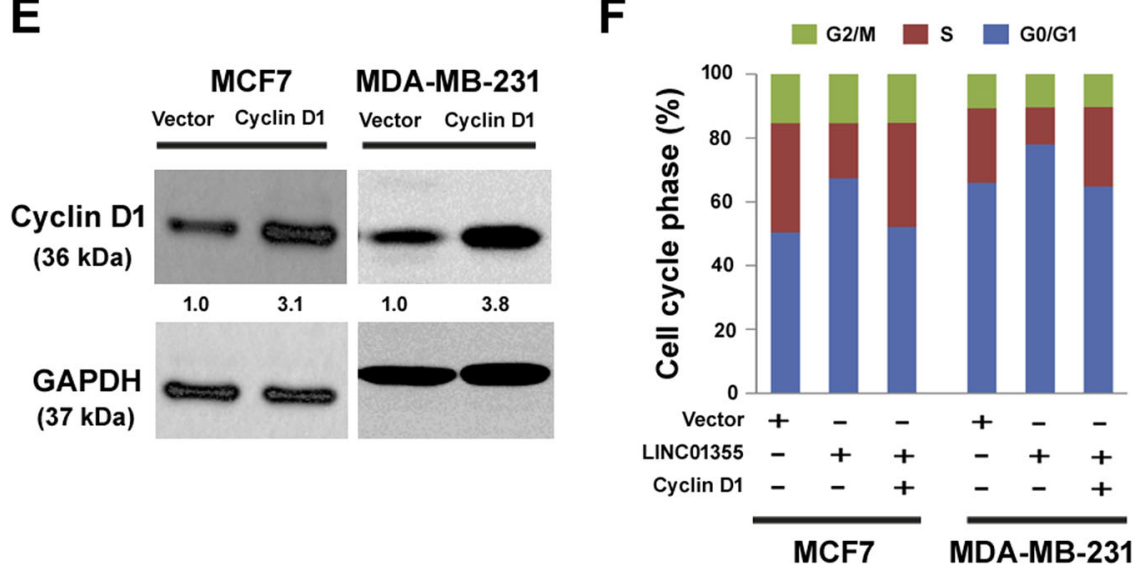

Fig. 3 LINC01355 induces cell cycle arrest at the G0/G1 phase by repressing CCND1 expression. a, b Flow cytometric analysis of cell cycle progression in MCF7 and MDA-MB-231 cells transfected with empty vector or LINC01355-expressing plasmid after PI staining. C qRT-PCR analysis of CCND1 mRNA expression in MCF7 and MDA-MB-231 cells transfected with indicated constructs. ${ }^{*}, P<0.05$ vs. vector-transfected cells. d, e Western blot analysis of cyclin D1 protein levels in MCF7 and MDA-MB-231 transfected with indicated constructs. Numbers represent fold change in protein levels. $\mathbf{f}$ Flow cytometric analysis of cell cycle progression in MCF7 and MDA-MB-231 cells transfected with indicated constructs 

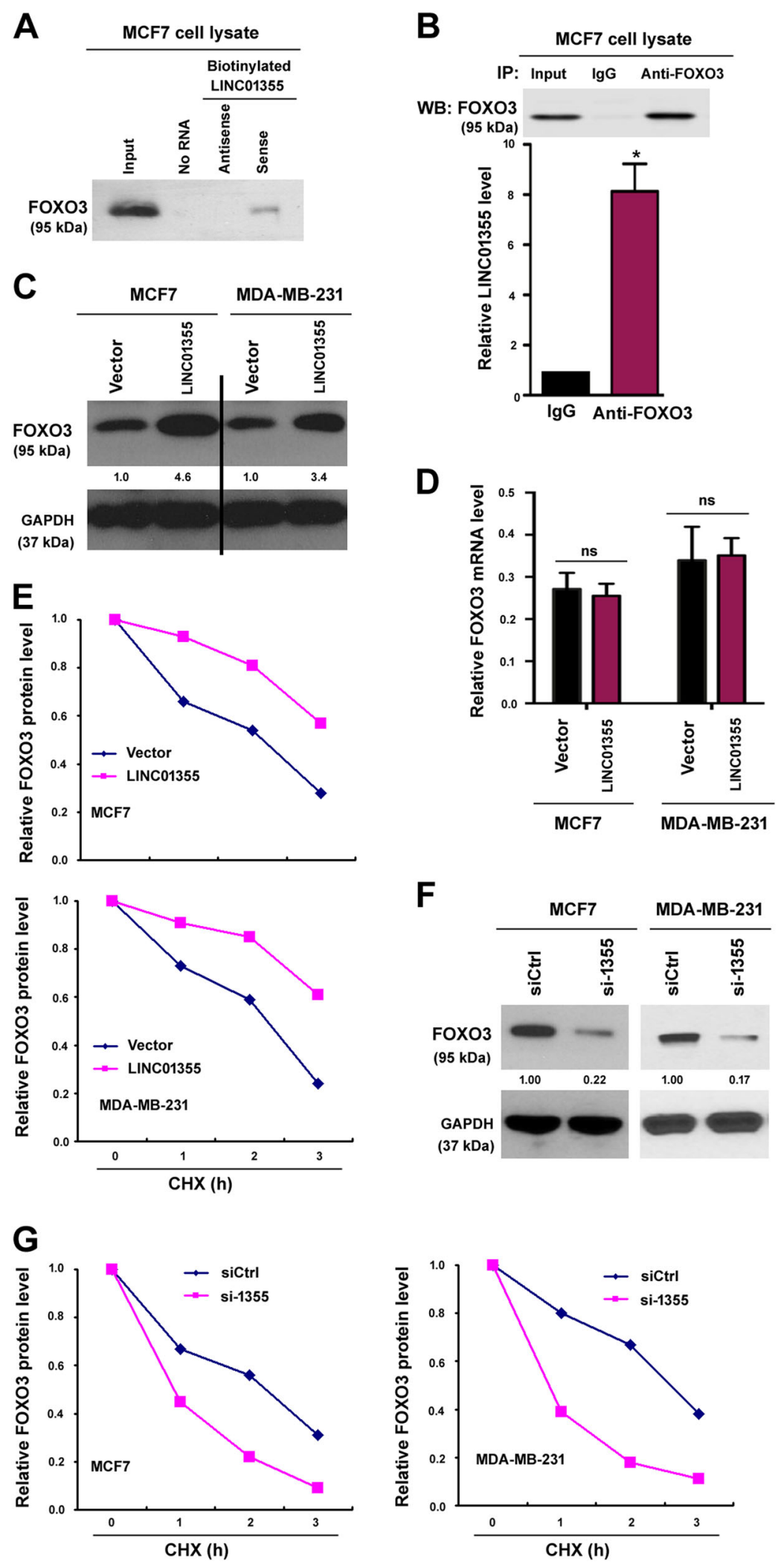

Fig. 4 (See legend on next page.) 
(see figure on previous page)

Fig. 4 LINC01355 interacts with and stabilizes FOXO3 protein. a RNA pull-down assay. Western blot analysis confirmed that FOXO3 protein was pulled down by biotinylated LINC01355 sense transcript but not by antisense transcript. b RIP assay revealed that LINC01355 was detected in FOXO3 immunoprecipitates from MCF7 cells. Upper, western blot analysis of FOXO3 immunoprecipitates. ${ }^{*}, P<0.05$ vs. control lgG immunoprecipitates. c Western blot analysis of FOXO3 protein levels in MCF7 and MDA-MB-231 transfected with indicated constructs. Numbers represent fold change in protein levels. $\mathbf{d}$ qRT-PCR analysis of FOXO3 mRNA expression in MCF7 and MDA-MB-231 cells transfected with indicated constructs. ns = no significance. e Western blot analysis of $\mathrm{FOXO} 3$ protein levels at different time points in transfected cells after treatment with $50 \mu \mathrm{m}$ cycloheximide. f Western blot analysis of FOXO3 protein levels in MCF7 and MDA-MB-231 transfected with indicated constructs. Numbers represent fold change in protein levels. $\mathbf{g}$ Western blot analysis of FOXO3 protein levels at different time points in transfected cells after treatment with $50 \mu \mathrm{m}$ cycloheximide
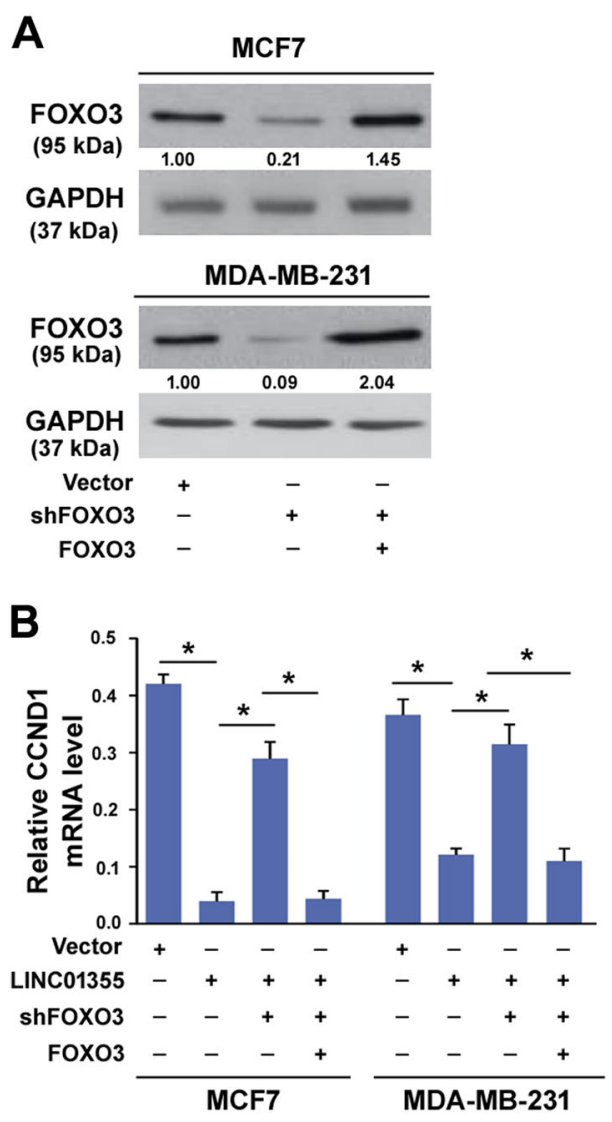

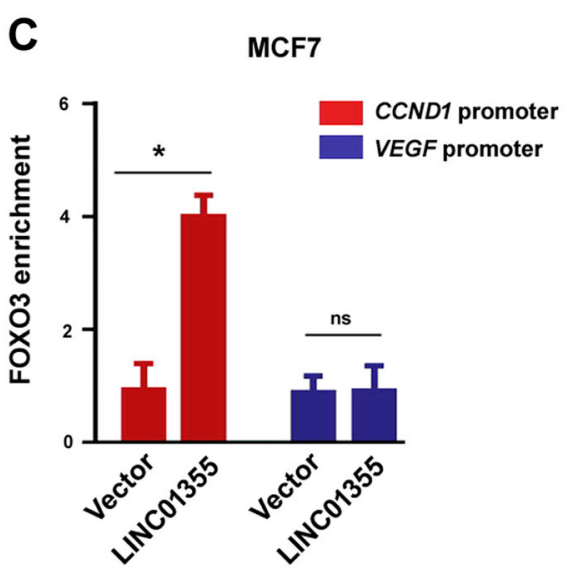

D MDA-MB-231

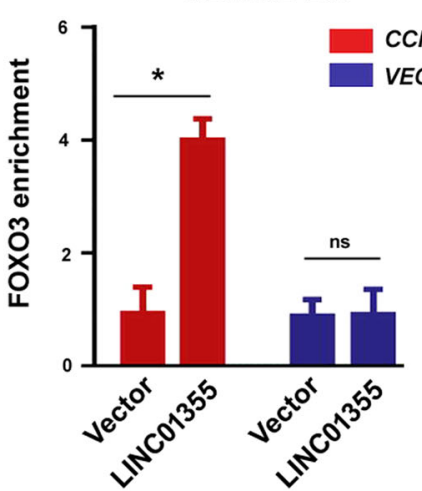

Fig. 5 LINC01355 promotes FOXO3-mediated transcriptional repression of CCND1. a Western blot analysis of FOXO3 protein levels in MCF7 and MDA-MB-231 transfected with indicated constructs. Numbers represent fold change in protein levels. $\mathbf{b}$ qRT-PCR analysis of CCND1 mRNA expression in MCF7 and MDA-MB-231 cells transfected with indicated constructs. ${ }^{*}, P<0.05$. $\mathbf{c}$, $\mathbf{d}$ ChIP assays were performed in MCF7 and MDA-MB231 transfected with indicated constructs to detect FOXO3 enrichment at the CCND1 promoter. The VEGF promoter was used as a control. ${ }^{*} P<0.05$; ns, no significance

together, we propose that LINC01355-induced downregulation of CCND1 depends on FOXO3 activity.

\section{Increased FOXO3 level is responsible for LINC01355-} induced tumor suppression

We next investigated the role of FOXO3 in the growth suppression induced by LINC01355. As shown in Fig. 6a and b, LINC01355-mediated inhibition of breast cancer cell proliferation and clonogenicity were reversed by knockdown of FOXO3. Analysis of cell cycle distribution showed that depletion of FOXO3 allowed LINC01355overexpressing MCF7 cells to re-enter into cell cycle (Fig. 6c). In vivo studies confirmed that FOXO3 deficiency abolished the suppression of MCF7 xenograft tumor 

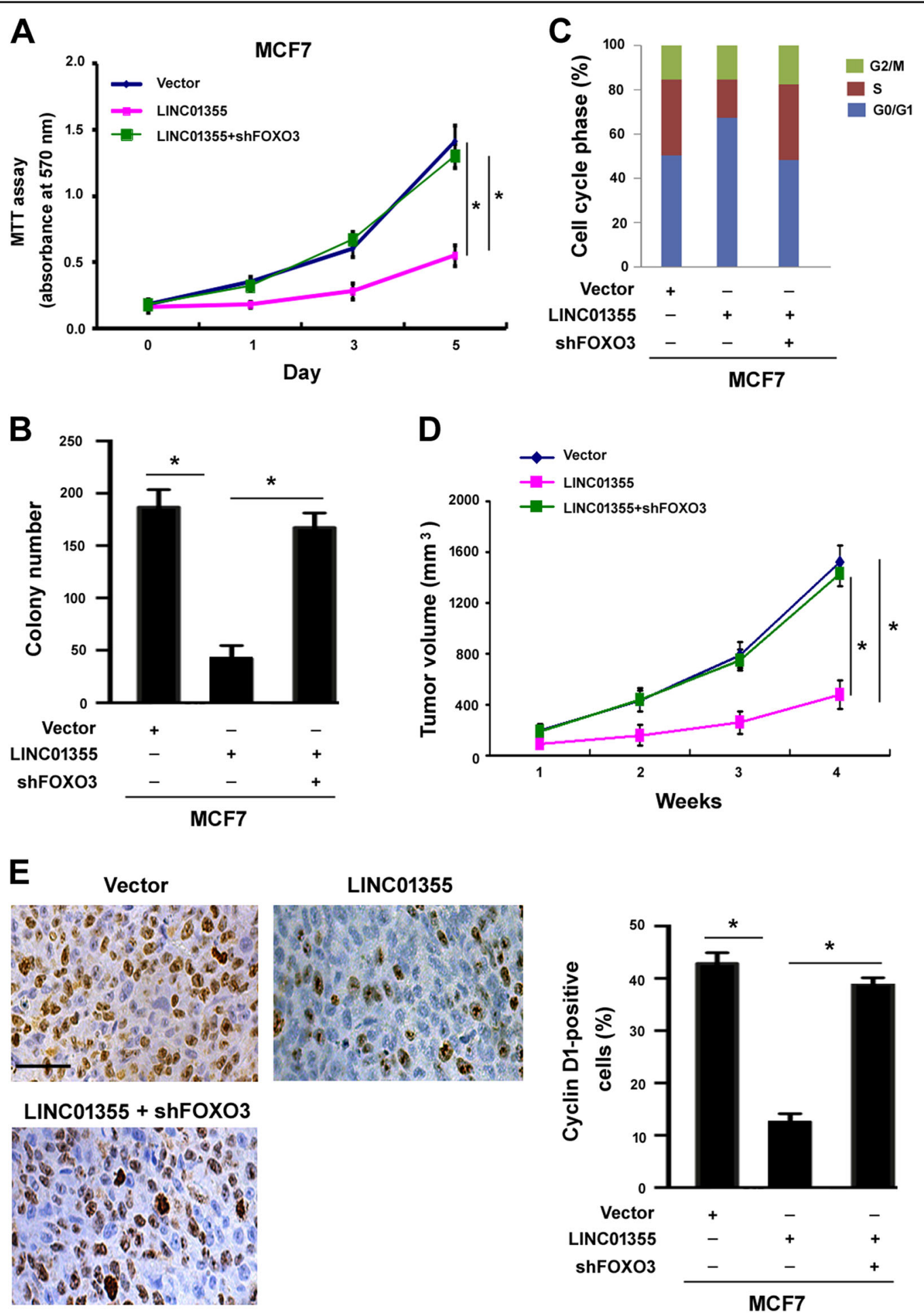

Fig. 6 Increased FOXO3 level is responsible for LINC01355-induced tumor suppression. a MCF7 cells transfected with indicated constructs were tested for proliferation at different time points by the MTT assays. b Colony formation assays were performed in MCF7 cells transfected with indicated constructs. c Flow cytometric analysis of cell cycle progression in MCF7 cells transfected with indicated constructs. d MCF7 cells were stably transfected with indicated constructs and inoculated into nude mice. Growth curves were plotted based on tumor volume. e Representative images of cyclin D1 immunostaining in tumor samples. Scale bar $=60 \mu \mathrm{m}$. d Quantification of cyclin D1 positive cells for each group. ${ }^{*} P<0.05$ 


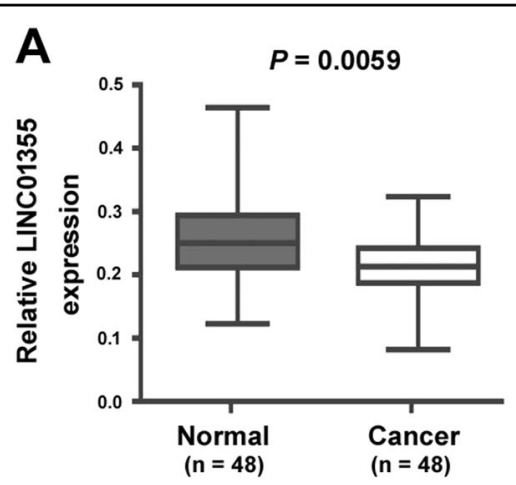

B

Correlation of LINC 01355 expression with clinical features
\begin{tabular}{|c|l|l|l|}
\hline Parameter & LINC01355 expression & $P$ \\
\hline & $\begin{array}{l}\text { Low } \\
(\mathbf{n}=24)\end{array}$ & $\begin{array}{l}\text { High } \\
(\mathbf{n}=\mathbf{2 4})\end{array}$ & \\
\hline Tumor size, cm & & & 0.0171 \\
\hline$<5$ & 11 & 19 & \\
\hline$\geq 5$ & 13 & 5 & 0.0079 \\
\hline TNM stage & & & \\
\hline I+II & 5 & 14 & \\
\hline III+IV & 19 & 10 & \\
\hline
\end{tabular}

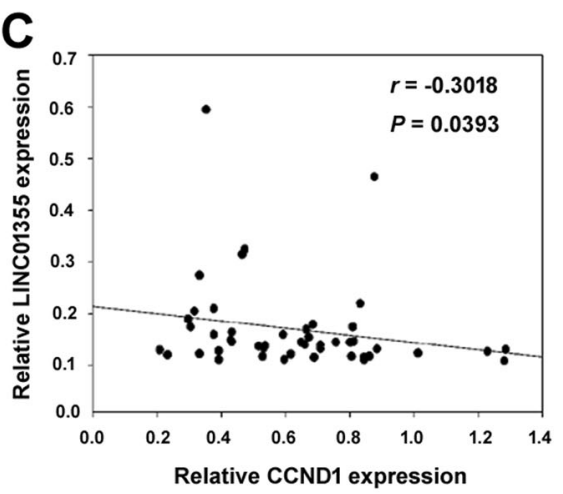

D LINC01355 interaction with FOXO3 protein

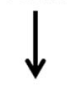

Stabilization of $\mathrm{FOXO} 3$ protein

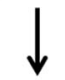

FOXO3 binding to CCND1 promoter

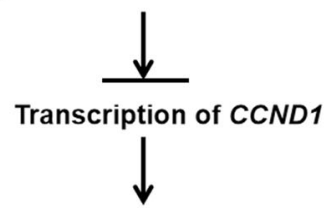

Breast cancer growth
Fig. 7 LINC01355 expression is correlated with clinical features and CCND1 mRNA levels in breast cancer. a qRT-PCR analysis of LINC01355 expression in paired breast cancer tissues and normal breast tissues. b Downregulation of LINC01355 was significantly correlated with larger tumor size and advanced clinical stage of breast cancer. c A negative correlation was detected between LINC01355 and CCND1 expression in breast cancer tissues. $\mathbf{d}$ Schematic illustration for the mechanisms by which LINC01355 interacts with FOXO3 to suppress breast cancer growth

growth induced by LINC01355 (Fig. 6d). Immunohistochemical analysis of cyclin D1 demonstrated that depletion of FOXO3 relieved the inhibition of cyclin D1 caused by LINC01355 (Fig. 6e). These observations suggest that FOXO3 is required for LINC01355-induced anticancer activity.

\section{LINC01355 expression is correlated with clinical features and CCND1 mRNA levels in breast cancer}

Next, we evaluated the expression and clinical significance of LINC01355 in breast cancer. We found that LINC01355 was significantly downregulated in breast cancer tissues relative to normal breast tissues $(P=$ 0.0059; Fig. 7a). Downregulation of LINC01355 was significantly correlated with larger tumor size $(P=0.0171)$ and advanced clinical stage $(P=0.0079)$ of breast cancer (Fig. 7b). However, the expression status of LINC01355 was not associated with age, lymph node metastasis, ER, or PR status (data not shown). The Spearman correlation analysis demonstrated a negative correlation between LINC01355 and CCND1 expression in breast cancer tissues $(r=-0.3018, P=0.0393$; Fig. 7c).

\section{Discussion}

The $1 \mathrm{p} 36$ locus is frequently deleted in breast cancer ${ }^{7}$, suggesting its involvement in cancer development and progression. Previously, an array of protein-coding tumor suppressor genes localized at the region have been identified $^{5,6}$. For example, CHD5 is downregulated and shows growth-suppressive activity in breast cancer ${ }^{26}$. Similarly, PER3 and RIZ1 exert inhibitory effects on aggressive phenotypes of breast cancer cells ${ }^{5,27}$. In this work, we focus on lncRNAs embedded in the 1p36 locus. We demonstrate that LINC01355 expression is underexpressed in breast cancer tissues and cell lines compared with their normal equivalents. Clinically, LINC01355 downregulation is significantly associated with tumor size and TNM stage in breast cancer. Functionally, LINC01355 knockdown renders a proliferation advantage to breast cancer cells, whereas ectopic expression of LINC01355 suppresses the proliferation and colony formation of breast cancer cells. In vivo data further validate the suppressive activity of LINC01355 in breast cancer tumorigenesis. LINC01355 overexpression leads to 
reduced proliferation and increased apoptosis in MCF7 xenograft tumors. Taken together, we provide first evidence that LINC01355 acts as a tumor suppressor in breast cancer.

Our data further demonstrate that LINC01355 overexpression causes a cell cycle arrest at the G0/G1 phase. Biochemically, LINC01355 overexpression inhibits the expression of cyclin D1 in breast cancer cells. Cyclin D1 is regarded as a key regulator of G1/S cell cycle transition through via the ability to bind and activate CDK4 and CDK6 kinases ${ }^{28}$. We found that overexpression of cyclin D1 reverses the accumulation of cells at the G0/G1 phase caused by LINC01355. In agreement with the in vitro findings, there is an inverse correlation between LINC01355 and CCND1 expression. These results suggest that downregulation of cyclin D1 is an important mechanism responsible for the growth-suppressive activity of LINC01355.

Several mechanisms have been proposed to be involved in the regulation of cyclin $\mathrm{D} 1^{29,30}$. For example, the deubiquitylase ubiquitin-specific peptidase 22 posttranslationally regulates cyclin D1 expression by preventing proteasome-mediated degradation ${ }^{29}$. LIN28A has been shown to promote the expression of cyclin D1 by inhibiting the production of let- $7^{30}$. A number of transcription factors such as FOXO3 participate in the transcriptional regulation of $C C N D 1^{21}$. Here, we provide evidence for the association between LINC01355 and FOXO3 protein in breast cancer cells. LINC01355 overexpression increases the level of FOXO3 protein but not mRNA, implying the post-transcriptional regulation of FOXO3. Indeed, the interaction with LINC01355 enhances FOXO3 protein stability, as evidenced by prolonged half-life of FOXO3 protein. Besides increased protein stability, LINC01355 selectively enhances the binding of FOXO3 protein to the promoter of CCND1. As a negative control, LINC01355 fails to impact the FOXO3 binding to $V E G F$ promoter. These data collectively point toward that LINC01355 acts as a stabilizer of FOXO3 and downregulates CCND1 through enhancement of FOXO3mediated transrepression. Consistent with the finding that knockdown of FOXO3 abrogates LINC01355induced downregulation of CCND1, FOXO3 depletion reverses the inhibitory effect of LINC01355 on breast cancer cell proliferation and tumorigenesis. Therefore, we propose that the FOXO3/CCND1 axis plays an essential role in LINC01355-mediated tumor suppression (Fig. 7d).

It has been previously reported that the USP9x deubiquitinase is involved in the stabilization of FOXO3 protein $^{31}$. We speculate that LINC01355 binding likely promotes the association between FOXO3 and deubiquitinases, thus reducing proteasomal degradation of FOXO3. Ongoing studies are conducted to identify the key mediator for LINC01355-mediated stabilization of
FOXO3. In addition, it remains to be determined whether LINC01355 can exert its tumor-suppressing activity in other cancers besides breast cancer.

In conclusion, our study identifies LINC01355 as a novel tumor suppressor. Downregulation of LINC01355 contributes to aggressive phenotype of breast cancer. Reexpression of LINC01355 suppresses the growth and tumorigenesis of breast cancer cells, which involves the stabilization of FOXO3 and enhancement of FOXO3mediated transrepression of CCND1. Therefore, strategies to induce LINC01355 expression may have therapeutic potential in breast cancer.

\section{Materials and methods \\ Cell lines}

All cell lines were obtained from the Type Culture Collection of the Chinese Academy of Sciences (Shanghai, China). The cells were cultured in RPMI-1640 medium (Lonza, Verviers, Belgium) supplemented with 10\% fetal bovine serum (Sigma-Aldrich, St. Louis, MO, USA), penicillin $(100 \mathrm{U} / \mathrm{ml})$, and streptomycin $(100 \mu \mathrm{g} / \mathrm{ml})$. Cell lines were validated to be free of mycoplasma.

\section{Quantitative real-time PCR (qRT-PCR) analysis}

Total RNA was isolated from cell lines and tissue samples using Trizol (Invitrogen, Carlsbad, CA, USA) following manufacturer's instructions. For qRT-PCR, RNA was reverse transcribed using the High-Capacity CDNA Reverse Transcription Kit (Invitrogen). PCR was performed using the Power SYBR Master Mix (Applied Biosystems, Foster City, California, USA). The primers used are listed in Table 1. Relative expression of each target gene was normalized to GAPDH mRNA level and calculated by the $2^{-\Delta \Delta \mathrm{Ct}}$ method $^{32}$.

\section{Gene knockdown by RNA interfering technology}

For gene knockdown experiments, gene-specific siRNA or short hairpin RNA were synthesized by Hanyu Biotech (Beijing, China). Target sequences are listed in Table 1. Cells were transfected using Lipofectamine 3000 (Invitrogen) following the manufacturer's instructions. Twenty-four hours after transfection, cells were subjected to further experiments. FOXO3 stably knockdown MCF7 cells were generated after puromycin selection. Knockdown efficiency was determined using qRT-PCR or western blot analyses.

\section{Plasmid construction and transfection}

The plasmids encoding LINC01355 (NR_110616), CCND1 (NM_053056), and FOXO3 (NM_001455) were purchased from Hanyu Biotech. The inserts were confirmed by sequencing. The plasmids were transfected into cells using Lipofectamine 3000. For generation of stable cell lines, transfected cells were selected with $800 \mu \mathrm{g} / \mathrm{ml}$ G418 (Sigma-Aldrich). 
Table 1 List of oligonucleotides used in the study

\begin{tabular}{|c|c|}
\hline qRT-PCR primers & Sequence \\
\hline \multirow[t]{2}{*}{ LINC01342 } & Forward: GTTGACTTGTTCAGGCACA \\
\hline & Reverse: GTCCTCCAAAGACGAGAACAG \\
\hline \multirow[t]{2}{*}{ PINK1-AS } & Forward: GAGCCTGTTGGCCAACAAAGT \\
\hline & Reverse: GTTGAGACTGTGTTAACAGATG \\
\hline \multirow[t]{2}{*}{ PIK3CD-AS1 } & Forward: AGCAGTAAACCTTCCCCTCC \\
\hline & Reverse: TCTTGAACCCCACCAGACTC \\
\hline \multirow[t]{2}{*}{ LINC01772 } & Forward: CTTACTTGAGGTTAGGAGTTC \\
\hline & Reverse: GTACAGTGGCATGATCTCAG \\
\hline \multirow[t]{2}{*}{ LINC01355 } & Forward: CTGCTCTAGCCCCTAAAGATAG \\
\hline & Reverse: GGATTCCAAATGACACATTCCT \\
\hline \multirow[t]{2}{*}{ CCND1 } & Forward: GCTGCGAAGTGGAAACCATC \\
\hline & Reverse: CCTCCTTCTGCACACATTTGAA \\
\hline \multirow[t]{2}{*}{$\mathrm{FOXO3}$} & Forward: TCTACGAGTGGATGGTGCGTT \\
\hline & Reverse: CGACTATGCAGTGACAGGTTGTG \\
\hline \multirow[t]{2}{*}{ GAPDH } & Forward: ACGGATTTGGTCGTATTGGGC \\
\hline & Reverse: TTGACGGTGCCATGGAATTG \\
\hline \multicolumn{2}{|l|}{ ChIP qPCR primers } \\
\hline \multirow[t]{2}{*}{ CCND1 } & Forward: GAAATCCCTITAACTITTAG \\
\hline & Reverse: GAATCTCAGCGACTGCATCT \\
\hline \multirow[t]{2}{*}{ VEGF } & Forward: TCCGGGTITTATCCCTCTTC \\
\hline & Reverse: TCTGCTGGTTTCCAAAATCC \\
\hline \multicolumn{2}{|l|}{ shRNA } \\
\hline shFOXO3 & CCGGCATGTTCAATGGGAGCTTGGACTCGAGTCCAAGCTCCCATTGAACATG TITाT \\
\hline \multicolumn{2}{|l|}{ siRNA } \\
\hline siCtrl & AGCUUCAUAAGGCGCAUAG \\
\hline si-1355 \#1 & CCUCAACUCCUGCUUCCAA \\
\hline si-1355 \#2 & CCAAUUGCAUAAUAUGGUU \\
\hline si-1355 \#3 & CUCACACUGAAUUGAUCAA \\
\hline
\end{tabular}

siCtrl control siRNA, si-1355 LINC01355-targeing siRNA

\section{Western blot analysis}

Cells were lysed in ice-cold buffer $(50 \mathrm{mM}$ Tris- $\mathrm{HCl}, \mathrm{pH}$ 7.4, 150 mM NaCl, 1 mM EDTA, 1 mM EGTA, 1\% NP-40, $0.1 \%$ sodium dodecyl sulfate (SDS), $1 \%$ triton $\mathrm{X}-100$ ) containing protease/phosphotase inhibitor cocktails (Sigma-Aldrich). Protein concentration was quantified using the BCA protein assay (Bio-Rad Laboratories, Hercules, CA, USA). Equal amounts of protein were separated by SDS-PAGE and transferred to nitrocellulose membranes. The membranes were incubated with $5 \%$ fat-free milk at room temperature for $1 \mathrm{~h}$ to block nonspecific binding, and probed with the primary antibodies recognizing cyclin D1, FOXO3, and GAPDH (Cell Signaling Technology, Inc., Beverly, MA, USA). After washing, the blots were incubated with peroxidase-labeled secondary antibodies (Cell Signaling Technology) and developed using an enhanced chemiluminescence detection kit (Amersham Biosciences, Inc., Piscataway, NJ, USA). The intensities of the bands were quantified by densitometry using Quantity One software (Bio-Rad Laboratories).

\section{Cell proliferation assay}

Cells were seeded onto 96 -well plates $\left(5 \times 10^{3}\right.$ cells/well $)$ and cultured for 1,3 , and 5 days. The 3-[4,5-dimethylthiazol-2-yl]-2,5 diphenyl tetrazolium bromide (MTT) solution (Sigma-Aldrich) was added to each well and incubated for $4 \mathrm{~h}$ at $37^{\circ} \mathrm{C}$ in a humidified atmosphere. 
The MTT formazan precipitate was dissolved in dimethyl sulfoxide. Absorbance was measured at $570 \mathrm{~nm}$. Cell growth curves were plotted.

\section{Colony formation assay}

Colony formation assay was performed as described previously $^{33}$. In brief, cells were plated in six-well plates (600 cell/well) and allowed to grow for an additional 12 days. The colonies were fixed in $70 \%$ ethanol and then stained with $0.5 \%$ crystal violet staining solution. The colonies were counted under a microscope.

\section{In vivo studies}

Female Balb/c nude mice (5-week-old) were used for xenograft experiments. MCF7 cells stably transfected with indicated constructs were subcutaneously inoculated into nude mice $\left(3 \times 10^{6}\right.$ cells/mouse). Tumor volume was determined every week for 4 weeks after cell injection. The animals were then sacrificed, and xenograft tumors were weighed. Xenograft tumor samples were formalinfixed, paraffin-embedded, and sectioned. Tissue sections were deparaffinized, rehydrated, and incubated with antiKi-67 and anti-cyclin D1 antibodies. Diaminobenzidine was used for colorimetric development.

\section{Flow cytometric analysis of cell cycle distribution}

Cells were fixed in ice-cold 70\% ethanol and treated with $50 \mu \mathrm{g} / \mathrm{mL}$ propidium iodide (Sigma-Aldrich) for $30 \mathrm{~min}$ in the dark. The cell cycle profile was assayed using a flow cytometer (BD Biosciences, San Jose, CA, USA).

\section{RNA pull-down assay}

RNA pull-down were performed as described previously $^{34}$. In brief, biotin-labeled LINC01355 (sense or antisense) was generated using the Biotin RNA Labeling Mix containing T7 RNA polymerase (Roche Diagnostics, Indianapolis, IN, USA) and incubated with MCF7 cellular lysates. Streptavidin agarose beads were added to retrieve protein

immunoprecipitates. LINC01355-immunoprecipitated proteins were subjected to mass spectrometric analysis and western blot analysis.

\section{RNA immunoprecipitation (RIP) assay}

RIP experiments were performed as described previously $^{35}$. In brief, MCF7 cells were lysed, and the lysates were immunoprecipitated with anti-FOXO3 antibody or control isotype IgG, followed by RNA recovery. The coprecipitated RNAs were detected by qRT-PCR.

\section{ChIP assay}

ChIP assay was performed as described previously ${ }^{36}$. In brief, cells transfected with empty vector or LINC01355expressing plasmids were cross-linked using $1 \%$ formaldehyde. Chromatin was isolated and sonicated to small fragments. Anti-FOXO3 antibodies were used to precipitate DNA-protein complexes. ChIP DNA was analyzed by qRT-PCR. The primers are listed in Table 1 .

\section{Determination of FOXO3 protein turnover}

Analysis of protein turnover was performed as described previously ${ }^{37}$. In brief, cells were transfected with empty vector or LINC01355-expressing plasmids and then treated with $50 \mu \mathrm{m}$ cycloheximide (Sigma-Aldrich) to inhibit protein synthesis. Cells were lysed at indicated time points and subjected to western blot analysis.

\section{Patients and samples}

Forty-eight breast cancer tissue samples and adjacent noncancerous breast tissues were collected from breast cancer patients who underwent surgical resection between 2016 and 2017 at our hospital. Diagnosis was confirmed by histology. No patient received pre-operative anticancer treatments. Tissue samples were snap-frozen in liquid nitrogen and stored at $-80^{\circ} \mathrm{C}$ prior to RNA isolation.

\section{Statistical analysis}

Differences among groups were analyzed by the Student's $t$ test, one-way analysis of variance, or Mann-Whitney $U$ test. The Spearman correlation analysis was used to analyze the relationship between LINC01355 and CCND1 expression in breast cancer tissues. The Chi-square test was used to analyze the associations of LINC01355 expression with pathologic features of breast cancer. $P<0.05$ was considered significant.

\section{Acknowledgements}

This study was supported by PUMC Youth Fund and the Fundamental Research Funds for the Central Universities (No. 3332015157), Capital Public Health Education, Beijing Science and Technology Program (No. Z171100000417028), Beijing Municipal Natural Science Foundation (Key program, No. 7191009), Natural Science Foundation of China (No. 81872160), Capital Public Health Education, Beijing Science and Technology Program (No. Z161100000116093), and CAMS Initiative for Innovative Medicine (No. 2016-12M-1-007)

\section{Author contributions}

B.A., J.W., Z.W. and Y.F. designed the study, analyzed the data, and prepared the manuscript. B.A., X.K., X.W., K.Z., X.Y. and J.Z. performed cellular and animal experiments and collected the data. Y.Q. and R.G. performed clinical examination. All authors read and approved the final manuscript.

Conflict of interest

None of the authors have any competing interests in the manuscript.

\section{Publisher's note}

Springer Nature remains neutral with regard to jurisdictional claims in published maps and institutional affiliations.

Supplementary Information accompanies this paper at (https://doi.org/ 10.1038/s41419-019-1741-8). 
Received: 27 January 2019 Revised: 16 May 2019 Accepted: 16 May 2019 Published online: 26 June 2019

\section{References}

1. Siegel, R. L., Miller, K. D. \& Jemal, A. Cancer statistics, 2018. CA Cancer J. Clin. 68 7-30 (2018)

2. Royce, M. et al. Everolimus plus endocrine therapy for postmenopausa women with estrogen receptor-positive, human epidermal growth factor receptor 2-negative advanced breast cancer: a clinical trial. JAMA Oncol. 4, 977-984 (2018).

3. Baselga, J. et al. Buparlisib plus fulvestrant versus placebo plus fulvestrant in postmenopausal, hormone receptor-positive, HER2-negative, advanced breast cancer (BELLE-2): a randomised, double-blind, placebo-controlled, phase 3 trial. Lancet Oncol. 18, 904-916 (2017).

4. Zhang, H. et al. Genome-wide association study identifies 1 p36.22 as a new susceptibility locus for hepatocellular carcinoma in chronic hepatitis B virus carriers. Nat. Genet. 42, 755-758 (2010).

5. Climent, J. et al. Deletion of the PER3 gene on chromosome 1 p36 in recurrent ER-positive breast cancer. J. Clin. Oncol. 28, 3770-3778 (2010).

6. Henrich, K. O., Schwab, M. \& Westermann, F. 1 p36 tumor suppression-a matter of dosage? Cancer Res. 72, 6079-6088 (2012).

7. Nagai, $H$. et al. Detection and cloning of a common region of loss of heterozygosity at chromosome $1 p$ in breast cancer. Cancer Res. 55, 1752-1757 (1995).

8. Henrich, K. O. et al. CAMTA1, a 1p36 tumor suppressor candidate, inhibits growth and activates differentiation programs in neuroblastoma cells. Cancer Res. 71, 3142-3151 (2011).

9. Lin, N. et al. Deletion or epigenetic silencing of AJAP1 on 1p36 in glioblastoma. Mol. Cancer Res. 10, 208-217 (2012).

10. Liu, Z. et al. CASZ1, a candidate tumor-suppressor gene, suppresses neuroblastoma tumor growth through reprogramming gene expression. Cell Death Differ. 18, 1174-1183 (2011).

11. Welch, C., Chen, Y. \& Stallings, R. L. MicroRNA-34a functions as a potential tumor suppressor by inducing apoptosis in neuroblastoma cells. Oncogene $\mathbf{2 6}$ 5017-5022 (2007).

12. Zampetaki, A., Albrecht, A. \& Steinhofel, K. Long non-coding RNA structure and function: is there a link? Front. Physiol. 9, 1201 (2018).

13. Liao, L. M. et al. Role of long noncoding RNA 799 in the metastasis of cervical cancer through upregulation of TBL1XR1 expression. Mol. Ther. Nucleic Acids 13, 580-589 (2018).

14. Dong, $\mathrm{H}$. et al. Long non-coding RNA SNHG14 induces trastuzumab resistance of breast cancer via regulating PABPC1 expression through $\mathrm{H} 3 \mathrm{~K} 27$ acetylation. J. Cell Mol. Med. 22, 4935-4947 (2018).

15. Wang, S. et al. LncRNA MIR100HG promotes cell proliferation in triple-negative breast cancer through triplex formation with p27 loci. Cell Death Dis. 9, 805 (2018).

16. Wang, R., Zhang, T., Yang, Z., Jiang, C. \& Seng, J. Long non-coding RNA FTH1P3 activates paclitaxel resistance in breast cancer through miR-206/ABCB1. J. Cell Mol. Med. 22, 4068-4075 (2018).

17. Luo, L. et al. LINC01638 IncRNA activates MTDH-Twist1 signaling by preventing SPOP-mediated C-Myc degradation in triple-negative breast cancer. Oncogene 37, 6166-6179 (2018).

18. Zhao, X. et al. CHP2 promotes cell proliferation in breast cancer via suppression of FOXO3a. Mol. Cancer Res. 16, 1512-1522 (2018).
19. Song, $\mathrm{Y}$. et al. Activation of $\mathrm{FOXO3a}$ reverses 5-Fluorouracil resistance in human breast cancer cells. Exp. Mol. Pathol. 105, 57-62 (2018).

20. Sisci, D. et al. The estrogen receptor $a$ is the key regulator of the bifunctional role of FoxO3a transcription factor in breast cancer motility and invasiveness. Cell Cycle 12, 3405-3420 (2013)

21. Liu, L. et al. High expression of Ras-related protein 1A promotes an aggressive phenotype in colorectal cancer via PTEN/FOXO3/CCND1 pathway. J. Exp. Clin. Cancer Res. 37, 178 (2018).

22. Karadedou, C. T. et al. FOXO3a represses VEGF expression through FOXM1dependent and -independent mechanisms in breast cancer. Oncogene 31, 1845-1858 (2012)

23. Dong, Q. et al. A novel hydrogen sulfide-releasing donor, HA-ADT, suppresses the growth of human breast cancer cells through inhibiting the PI3KIAKT/ mTOR and Ras/Raf/MEKJERK signaling pathways. Cancer Lett. 455, 60-72 (2019).

24. Fernández-Nogueira, P. et al. Histamine receptor 1 inhibition enhances antitumor therapeutic responses through extracellular signal-regulated kinase (ERK) activation in breast cancer. Cancer Lett. 424, 70-83 (2018).

25. Li, L. X. et al. Lysine methyltransferase SMYD2 promotes triple negative breast cancer progression. Cell Death Dis. 9, 326 (2018).

26. $\mathrm{Wu}, \mathrm{X}$. et al. Chromodomain helicase DNA binding protein 5 plays a tumor suppressor role in human breast cancer. Breast. Cancer Res. 14, R73 (2012).

27. $\mathrm{He}$, L. et al. RIZ1, but not the alternative RIZ2 product of the same gene, is underexpressed in breast cancer, and forced RIZ1 expression causes G2-M cell cycle arrest and/or apoptosis. Cancer Res. 58, 4238-4244 (1998).

28. O'Leary, B., Finn, R. S. \& Turner, N. C. Treating cancer with selective CDK4/6 inhibitors. Nat. Rev. Clin. Oncol. 13, 417-430 (2016).

29. Gennaro, V. J. et al. Control of CCND1 ubiquitylation by the catalytic SAGA subunit USP22 is essential for cell cycle progression through G1 in cancer cells. Proc. Natl. Acad. Sci. USA 115, E9298-E9307 (2018).

30. Li, N. et al. Lin-28 homologue A (LIN28A) promotes cell cycle progression via regulation of cyclin-dependent kinase 2 (CDK2), cyclin D1 (CCND1), and cell division cycle 25 homolog A (CDC25A) expression in cancer. J. Biol. Chem. 287 17386-17397 (2012).

31. Zheng, X. et al. Prolyl hydroxylation by EgIN2 destabilizes FOXO3a by blocking its interaction with the USP9x deubiquitinase. Genes Dev. 28, 1429-1444 (2014).

32. Livak, K. J. \& Schmittgen, T. D. Analysis of relative gene expression data using real-time quantitative PCR and the 2(-Delta Delta C(T)) method. Methods $\mathbf{2 5}$ 402-408 (2001).

33. Gao, J. et al. Cyclin $\mathrm{G} 2$ suppresses Wnt/ $\beta$-catenin signaling and inhibits gastric cancer cell growth and migration through Dapper1. J. Exp. Clin. Cancer Res. 37, 317 (2018).

34. Zhang, E. et al. A novel long noncoding RNA HOXC-AS3 mediates tumorigenesis of gastric cancer by binding to YBX1. Genome. Biol. 19, 154 (2018).

35. Chen, J. F. et al. STAT3-induced IncRNA HAGLROS overexpression contributes to the malignant progression of gastric cancer cells via mTOR signal-mediated inhibition of autophagy. Mol. Cancer 17, 6 (2018).

36. Sakurai, K., Reon, B. J., Anaya, J. \& Dutta, A. The IncRNA DRAIC/PCAT29 locus constitutes a tumor-suppressive nNexus. Mol. Cancer Res. 13, 828-838 (2015).

37. IzzO, F. et al. Progesterone receptor activation downregulates GATA3 by transcriptional repression and increased protein turnover promoting breast tumor growth. Breast Cancer Res. 16, 491 (2014). 